\title{
Effects of perceptual context on transsaccadic visual matching
}

\author{
MARTIN JÜTTNER \\ University of Munich, Munich, Germany
}

\begin{abstract}
Transsaccadic visual matching refers to the phenomenon in which presaccadic signals at the location of the saccade goal influence the visibility of postsaccadic test signals presented at the fovea (Jüttner \& Röhler, 1993). The present study uses variations of the perceptual context, provided by the structure of the presaccadic stimulus display, to investigate the influence of spatial information, decision factors, and visual attention on this form of transsaccadic information processing. The experiments yielded the following results: First, analysis of the data in terms of signal detection theory revealed that transsaccadic visual matching manifests in a shift of decision criterion $(\beta)$ rather than in a change of sensory sensitivity $\left(d^{\prime}\right)$. The criterion shift leads to a suppression of postsaccadic stimulus information that is incompatible with presaccadic processed information. Second, the matching effect strongly depends on the structure of the presaccadic display, which suggests that mechanisms of visual attention provide the spatial binding of pre- and postsaccadic stimulus information. Third, transsaccadic matching is phenomenologically tied to the execution of the saccade and effective during a postsaccadic period extending up to $160 \mathrm{msec}$ after termination of the eye movement. These findings indicate a form of context-sensitive evaluation process that could subsidize the maintenance of visual stability.
\end{abstract}

When we look at pictures or scan our visual environment, our eyes are almost permanently moving. Fast ballistic eye movements, or saccades, separate brief periods of time (fixations) during which the eyes remain almost stationary. During the saccade, vision is severely impaired. Saccadic suppression (see, e.g., Riggs, Merton, \& Morton, 1974; Volkman, Schick, \& Riggs, 1968) reduces visual sensitivity and suppresses the perception of the smeared retinal image during the eye movement. Moreover, sensitivity to displacement of targets decreases substantially so that motion and position information becomes severely degraded (Bridgeman, 1983; Bridgeman, Hendry, \& Stark, 1975). Acquisition of visual information therefore takes place mainly during the fixations, and the sequence of fixational pauses corresponds to a series of discrete retinal images. Even if we assume a stationary head and body, the retinal projections still differ from each other as the mapping of spatiotopic to retinal coordinates depends on the dynamic position of the eye. But despite these differences, the apparent position of objects in the world remains constant and our experience of the world is stable and continuous rather than being fragmented into individual glimpses.

To explain these phenomena it has often been assumed that the perceptual system constructs a representation

\footnotetext{
This study was conducted as part of M.J.'s PhD thesis at the Institute of Medical Optics, Department of Physics, University of Munich. It was supported by Grant RE 337/5-1 and JU 230/2-1 of Deutsche Forschungsgemeinschaft. M.J. thanks I. Rentschler and R. Röhler for helpful suggestions on earlier drafts of this manuscript. Correspondence should be addressed to M. Jüttner, Institute for Medical Psychology, University of Munich, Goethestrasse 31, D-80336 Munich, Germany (e-mail: martin@groucho.imp.med.uni-muenchen.de).
}

based on spatiotopic coordinates. Different versions of this type of solution exist (see, e.g., Bischof \& Kramer, 1968; Breitmeier, Kropfl, \& Julesz, 1982; Feldman, 1985), but their common ratio is the notion of a spatiotopic store that is linked to the retinal representation by a coordinate transformation, compensating for the translation of the retinal image caused by the eye movement. With each fixation the recalibrated retinal image should then superimpose with the current image and thus provide an update of the internal representation. This solution appears plausible since it readily explains the aforementioned qualities of stability and continuity of our visual experience. However, psychophysical evidence does not support this hypothesis. Its important prediction of a spatiotopic superposition, or integration, of pre- and postsaccadic information has been disproved in numerous studies (e.g., Bridgeman \& Mayer, 1983; Irwin, Yantis, \& Jonides, 1983; Jonides, Irwin, \& Yantis, 1983; Rayner \& Pollatsek, 1983).

The failure of the integration solution comes as no surprise if we consider that vision is far from being isotropic across the visual field. Most saccades are in the range of $1^{\circ}$ to $5^{\circ}$, but even for small saccades, the anisotropy of the retina poses a constraint that cannot be neglected. Under photopic conditions, performance of extrafoveal vision is severely degraded with respect to foveal vision. In part these deficits can be explained by cortical sampling, which becomes sparser with increasing eccentricity and thus can in principle be compensated for by simply enlarging the stimuli appropriately (Koenderink, Bouman, Bueno de Mesquita, \& Slappendel, 1978; Rovamo \& Virsu, 1979). However, recent experimental evidence indicates that for complex stimuli and recognition tasks, performance cannot always be renormalized by a rescaling operation (Strasburger, Harvey, \& Rentschler, 1991; Strasburger, Rentschler, 
\& Harvey, 1994). Employing a probabilistic classification technique, we have recently demonstrated (Jüttner \& Rentschler, 1996; Rentschler, Jüttner, \& Caelli, 1994) that the internal representations of foveal and extrafoveal vision seem to be incommensurable in the sense that extrafoveal pattern representations are characterized by a lower perceptual dimension whereas foveal representations are not. More specifically, it was shown that the representations generated in foveal vision are complete in the sense that they employ the same number of perceptual dimensions as there are feature dimensions in the underlying physical feature space. In contrast, the representations of extrafoveal vision appear to be incomplete since their perceptual dimension is systematically reduced. A low-level integration of information would then appear to be of little benefit since it would imply a superposition of images that originate from foveal and extrafoveal parts of the retina and that therefore are perceptually not isomorphic.

But a low-level image integration represents merely one possible manifestation of cooperation across saccades. Instead it could be conceived that such processes occur on a more abstract, semantically oriented stage of analysis within the visual system. There is in fact evidence for a facilitation of perception of a stimulus if the same stimulus has been presented in a peripheral location before the saccade. Such priming effects have been found for the naming times of words (McConkie \& Zola, 1979; Rayner, McConkie, \& Zola, 1980) and pictures (Pollatsek, Rayner, \& Collins, 1984; Pollatsek, Rayner, \& Henderson, 1990). For words, this parafoveal preview benefit depends on whether the first two or three letters of preview and target are identical. Interestingly, the effect does not rely on physical identity because it remains unaffected if the case of all letters is changed from one fixation to the next. Rayner et al. have explained their findings by assuming that some abstract letter identity code is extracted during the first fixation and integrated with information of the postsaccadic target. With pictures, on the other hand, a parafoveal preview benefit also occurs with visually similar but semantically dissimilar pictures. This suggests that the parafoveal preprocessing must in part be based on feature-based representations rather than on purely nonvisual categorical concepts. With both types of stimuli, however, facilitation seems not to be directly linked to oculomotor activity, since priming effects also occur if, with stationary eyes, the saccade-evoked stimulus sequence on the retina is mimicked by an appropriate displacement of the stimuli (Pollatsek et al., 1990; Rayner et al., 1980). Irwin (1992) has related these facilitation effects to general-purpose memory mechanisms rather than assuming a specialized transsaccadic buffer.

Alternatively, one can reconsider the functional role of foveal and extrafoveal vision in the context of saccadic eye movements. Hayhoe, Lachter, and Feldman (1991) have emphasized that what is ecologically needed is not an enhancement of visual processing of the current foveal image by a previous peripheral stimulus, but rather the combination of successive foveal views in order to relate successive views in a spatial reference frame. Their experiments involve a perceptual judgment task in which observers were required to classify three successively presented points as forming either an acute or an obtuse angle. Precise judgments were observed even when the eyes were allowed to move between successive dot presentations, especially when a stable visible landmark (such as a single point) was continuously present. This result shows that successive foveal views may be aligned with respect to common, peripherally acquired, visual features. Hayhoe et al. have also interpreted their findings as evidence for a strategy of visual matching that in combination with eye position information could account for visual stability. But because of the specific experimental paradigm, their conception of visual matching involves only foveal views, leaving the role of peripheral vision rather unspecific apart from providing some sort of spatial reference.

Indeed, there is evidence that transsaccadic matching also occurs between peripheral and foveal vision in the sense that parafoveally acquired presaccadic information influences the way foveally obtained postsaccadic information is processed. Jüttner and Röhler (1993) introduced a paradigm by which they measured how presaccadic signals at the location of the saccade goal influence the visibility of postsaccadic test signals presented at the fovea. The signals were Landolt rings of different orientations. When the orientations of the pre- and postsaccadic Landolt rings were different, the thresholds of the test signals were elevated by about $20 \%-25 \%$ relative to those at a static control condition. When the orientations were identical, no such elevation occurred. These results can be interpreted in terms of a matching strategy in which foveally acquired ambivalent stimulus information is interpreted in a way that preserves compatibility with information that has been presaccadically obtained at the location of the saccade goal. The transsaccadic interference appeared to be a phenomenon linked to the active execution of the eye movement. Furthermore, the effect proved to be robust against a spatial decorrelation introduced by making the pre- and postsaccadic Landolt rings of different sizes, suggesting that the semantic content of the signal - that is, the orientation of the gap of the Landolt ring -is the critical parameter, rather than physical features based on a "pixel-wise" representation.

The following investigation aimed at clarifying some of the constraints of transsacccadic matching by manipulating the perceptual context provided by the structure of the presaccadic stimulus display. The goal was to elucidate the roles of (1) spatial information, by exploring the degree of invariance of this phenomenon in relation to different types of spatial decorrelation; (2) decision factors, by evaluating the results in terms of signal detection theory; and (3) of visual attention, by modifying the presaccadic stimulus configuration. 


\section{EXPERIMENTS 1 AND 2}

\section{Method}

Subjects. Four observers (A.T., M.H., M.J., S.D.) with normal or corrected-to-normal vision participated. Apart from Observer M.J., all subjects were completely naive with respect to the aim of the study.

Apparatus. Stimulus generation and presentation were controlled by a color pattern generator (see Gerber, 1987, for details) that permits the generation and manipulation of 2-dimensional $256 \times$ $256 \times 10$ bit patterns in real time at a frame rate of $50 \mathrm{~Hz}$. Stimuli were presented on a Barco CDCT 6151 monitor (cathode ray tube Hitachi 510 CKB 22; phosphor P22 with $10 \%$ decay rate of less than $1 \mathrm{msec}$, Skolnik, 1970). The mean luminance of the screen was adjusted to $20 \mathrm{~cd} / \mathrm{m}^{2}$. The modulation of the signals presented on the screen did not exceed $6 \%$ of the mean luminance. The screen subtended $28^{\circ} \times 21^{\circ}$ of visual angle at the viewing distance of $0.8 \mathrm{~m}$. Eye movements were recorded using an infrared scleral-reflectance technique with a horizontal resolution of $0.1^{\circ}$. The sampling rate for the eye position signal was restricted to $50 \mathrm{~Hz}$ due to the synchronization given by the frame rate of the pattern generator. Saccade onset was determined by applying an eye position change criterion of $1^{\circ}$ in $20 \mathrm{msec}$. Stimulus presentation, eye movement tracking, and response acquisition were controlled by a Digital Equipment Corporation PDP-11/24 microcomputer.

Stimuli. The stimuli were Landolt rings of four possible orientations: $0^{\circ}, 90^{\circ}, 180^{\circ}$, and $270^{\circ}$ (Figure 1). By definition, both line width and gap width equal 0.2 times the outside diameter of the Landolt ring. The standard viewing size of all rings was $1.7^{\circ}$. For Experiment 2, the parafoveally presented signals were randomly rescaled with scaling factors of $0.8,1.0,1.5$, and 2.0. Parafoveally presented Landolt rings had contrast values of $0.16 \mathrm{log}$ units so that they could be easily identified in peripheral vision. The foveally presented test stimuli were presented at a weak suprathreshold contrast level of about $0.04 \mathrm{log}$ units, corresponding to an overall identification accuracy of $60 \%-70 \%$ in the employed four-alternative forced-choice paradigm (see next section). Small black dots of $0.1^{\circ}$ diameter served as fixation points.

Procedure. Observers viewed the screen monocularly with the left eye. Their heads were fixed with a bite bar. All room lights in the experimental area were extinguished during the experimental sessions.

The two experimental conditions applied in the experiments were all derived from a common standard paradigm introduced by Jüttner and Röhler (1993). The stimulus sequence of this standard paradigm is illustrated in Figure 2. A trial started with the observer fixating a dot in the center of the monitor. The cross in Figure 2 symbolizes the subject's eye position and does not belong to the frame itself. At a distance of $3^{\circ}$ to the right of the initial fixation, the presaccadic stimulus, which consisted of a Landolt ring with randomly selected orientation, was presented. When the observer had achieved fixation, he/she pressed a button. After a randomized delay of $700-900 \mathrm{msec}$, the parafoveal stimulus disappeared. At the same time, the fixation dot jumped to its position. The subject had to saccade to the fixation point within a time window of $200 \mathrm{msec}$. Both the eye movement and the preceding fixation period after the ready signal were checked for correct execution. Trials with fixation errors above $1^{\circ}$ or improper execution of the saccade were discarded. In this case, the subject was informed by an acoustical signal and the disqualified trial was repeated later. After a variable interstimulus interval (ISI) of $260-500 \mathrm{msec}$, the test signal was presented for $20 \mathrm{msec}$, centered at the new position of the fixation point. The orientation of the test stimulus was randomly selected from one of the four possible values. As noted, the contrast of the test stimulus was always weak suprathreshold $(0.04 \mathrm{log}$

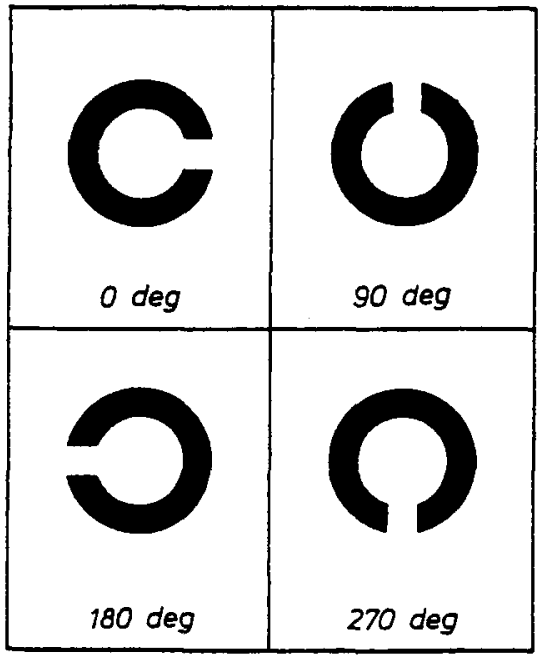

Figure 1. Landolt rings of gap orientations $\left(0^{\circ}, 90^{\circ}, 180^{\circ}\right.$, and $270^{\circ}$ ) serving as stimuli in the experiments.

units). This introduced a situation of decision uncertainty for the observer, who had to indicate the orientation by pressing a button on a keyboard. The keypress finished the trial and the next cycle started.

Experiments 1 and 2 differed from this standard paradigm only with respect to the appearance of the presaccadic display; the postsaccadic display (i.e., position and size of the test stimulus) remained unaltered. In Experiment 1, the size of the presaccadic Landolt ring could be scaled by a factor of $0.8,1.0,1.5$, or 2.0 with respect to the standard viewing size of $1.7^{\circ}$. In Experiment 2, the position of the presaccadic Landolt ring was chosen from one of the following values: $3^{\circ}$ left, $1.5^{\circ}$ right, $3.0^{\circ}$ right, and $4.5^{\circ}$ right with respect to the center of the screen.

Each trial in Experiments 1 and 2 was characterized by three parameters: the orientation of the presaccadic signal, its size (Experiment 1) or location (Experiment 2), and the orientation of the test signal. Thus the total number of stimulus configurations amounted to $4 \times 4 \times 4=64$. The experiments were blocked into 320 trial sessions yielding five presentations per configuration. The data from 10 sessions were pooled for a database of 50 presentations per configuration.

Data analysis. Jüttner \& Röhler (1993) used different contrast levels for the test signals and performed a threshold analysis on their data. In the present investigation, there was only one contrast level employed for the test stimuli, a choice that was motivated by our intention to explore the influence of other parameters of the presaccadic signals without facing a combinatorial explosion of stimulus configurations. The idea was to make the test stimulus semantically ambiguous by using a weak suprathreshold contrast level and to explore the degree to which the interpretation of the test signal depended on the presaccadic stimulus display.

Our data therefore consisted of relative frequencies with which observers had correctly identified the orientation of the postsaccadic test signal. Measures of accuracy such as thresholds or percentage correct are known to be subject to the influence of both sensory and decision processes. In order to disentangle the influence of both factors, we analyzed the data in terms of signal detection theory (SDT, Green \& Swets, 1974). Essentially, this approach involves a transformation of hit rate and false alarm rate into a detection accuracy measure $\left(d^{\prime}\right)$ and a decision bias measure $(\beta)$. As- 


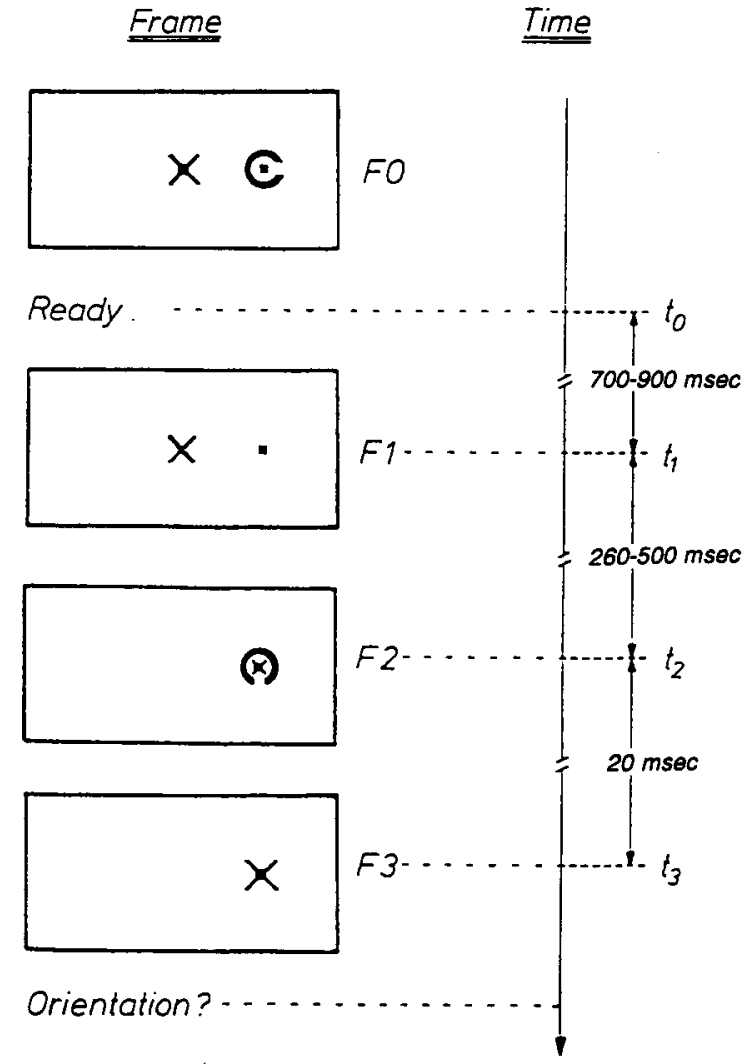

Figure 2. Stimulus sequence of the standard paradigm underlying Experiments 1 and 2. The subject started to fixate a dot in the center of the monitor. At a distance of $3^{\circ}$ of visual angle to the right, the presaccadic stimulus was presented. It consisted of a Landolt ring with randomly selected orientation. After the observer had signaled his/her readiness, the parafoveal stimulus disappeared after $700-900 \mathrm{msec}$. At the same time, the fixation dot jumped to its position. The subject had to saccade to the fixation point within a time window of $200 \mathrm{msec}$, and after an interstimulus interval of $260-500 \mathrm{msec}$, the test signal was presented. The test stimulus was centered at the location of the fixation dot, with randomly selected orientation. The subject had to indicate the orientation. Note that unlike the graphic illustration, the actual contrast of the test signal was only weak suprathreshold $(0.04 \mathrm{log}$ units), thus making the identification of the test signal ambivalent for the observer.

suming a signal detection model based on Gaussian probability distributions with equal variance, $d^{\prime}$ and $\beta$ are calculated from

$$
\begin{gathered}
d^{\prime}=z_{h r}-z_{f a r}, \\
x_{c}=-z_{f a r}, \\
\beta=-f_{s n}\left(x_{c}\right) / f_{n}\left(x_{c}\right),
\end{gathered}
$$

where $z_{h r}$ and $z_{f a r}$ are the $z$-score transforms of the hit rate and false alarm rate, respectively; $x_{c}$ is the critical value of the decision variable; and $f_{s n}\left(x_{c}\right)$ and $f_{n}\left(x_{c}\right)$ are the Gaussian probability densities $x_{c}$ for the target and nontarget distribution. $\mathrm{A} d d^{\prime}$ of 0.0 indicates no target detection accuracy; a $\beta$ value of 1.0 corresponds to an unbiased decision criterion.

For the practical application of this technique, the response data of each observer were divided into two classes, S and D, where S consisted of the responses for trials in which the orientations of the pre- and postsaccadic Landolt ring were the same, and D contained the response data for all trials in which the orientations were different. For each of the two response classes, the data were collapsed across the four response alternatives, yielding two relative frequencies concerning hit rate and false alarm rate, respectively. The two rates were transformed according to Equations 1-3 into a corresponding pair $\left(d^{\prime}, \beta\right)$. At this point it must be noted that without the subdivision into $\mathrm{S}$ and $\mathrm{D}$, a straight $\left(d^{\prime}, \beta\right)$ transformation of the total data would yield (and in fact always yielded in our experiments) a $\beta$ value of 1 . This is because of the forced-choice paradigm employed in the experiments, a method known to be criterion free as long as the observer exhibits no bias toward a particular response alternative. However, because the data were sorted according to the presaccadic stimulus history, this assertion is no longer valid for the individual Response Classes S and D. Here criterion effects may occur if they are caused by the presaccadic stimulus display, although they are not independent from each other and therefore have to be interpreted with respect to their common reference value of 1 .

\section{Results}

The first two experiments were conducted to determine the dependency of transsaccadic matching on the degree of spatial correlation between pre- and postsaccadic stimulus displays. Spatial decorrelation was varied in two different ways. Both affected only the appearance of the presaccadic stimulus display, whereas the postsaccadic stimulus display and therefore the saccade metrics remained unchanged. In Experiment 1, the presaccadic signal was rescaled with respect to the postsaccadic test signal by a factor of $0.8,1.0,1.5$, or 2.0 , whereas the center coordinates of both signals remained the same. In Experiment 2, the sizes of pre- and postsaccadic signal were equal, but the position of the presaccadic Landolt ring was shifted horizontally to one of the following values: $3^{\circ}$ left, $1.5^{\circ}$ right, $3.0^{\circ}$ right, and $4.5^{\circ}$ right with respect to the center of the screen.

For Experiment 1, Figure 3A shows the relative frequency for a correct identification of the orientation of the test stimulus for 4 subjects. The near-threshold contrast of the test signal makes the classification task for the observer ambiguous, and percentage correct values lie well below $100 \%$. However, the degree of ambiguity clearly depends on the stimulus history: For Condition D (i.e., different orientations of test stimulus and presaccadic prime signal), performance is distinctly reduced relative to Condition S (i.e., orientations of the two signals were identical). This confirms Jüttner \& Röhler's (1993) observation of decreased accuracy for the detection of transsaccadic modified signals. Moreover, this phenomenon obviously does not depend on the degree of spatiotopic congruence between pre- and postsaccadic stimulus displays. Rather, the effect turns out to be scale invariant across the entire range of size variations. This result extends an observation made already by Jüttner and Röhler, who applied a scale factor of 1.25 in their persistence-control-II experiment in order to avoid any pixel overlap of pre- and postsaccadic signal and to exclude phosphor persistence as a possible source of artifacts.

The question arises whether the reduced performance of Response Class D has to be attributed to sensory processes or is a consequence of an altered decision behavior. Figures $3 \mathrm{~B}$ and $3 \mathrm{C}$ show the results of the SDT analy- 

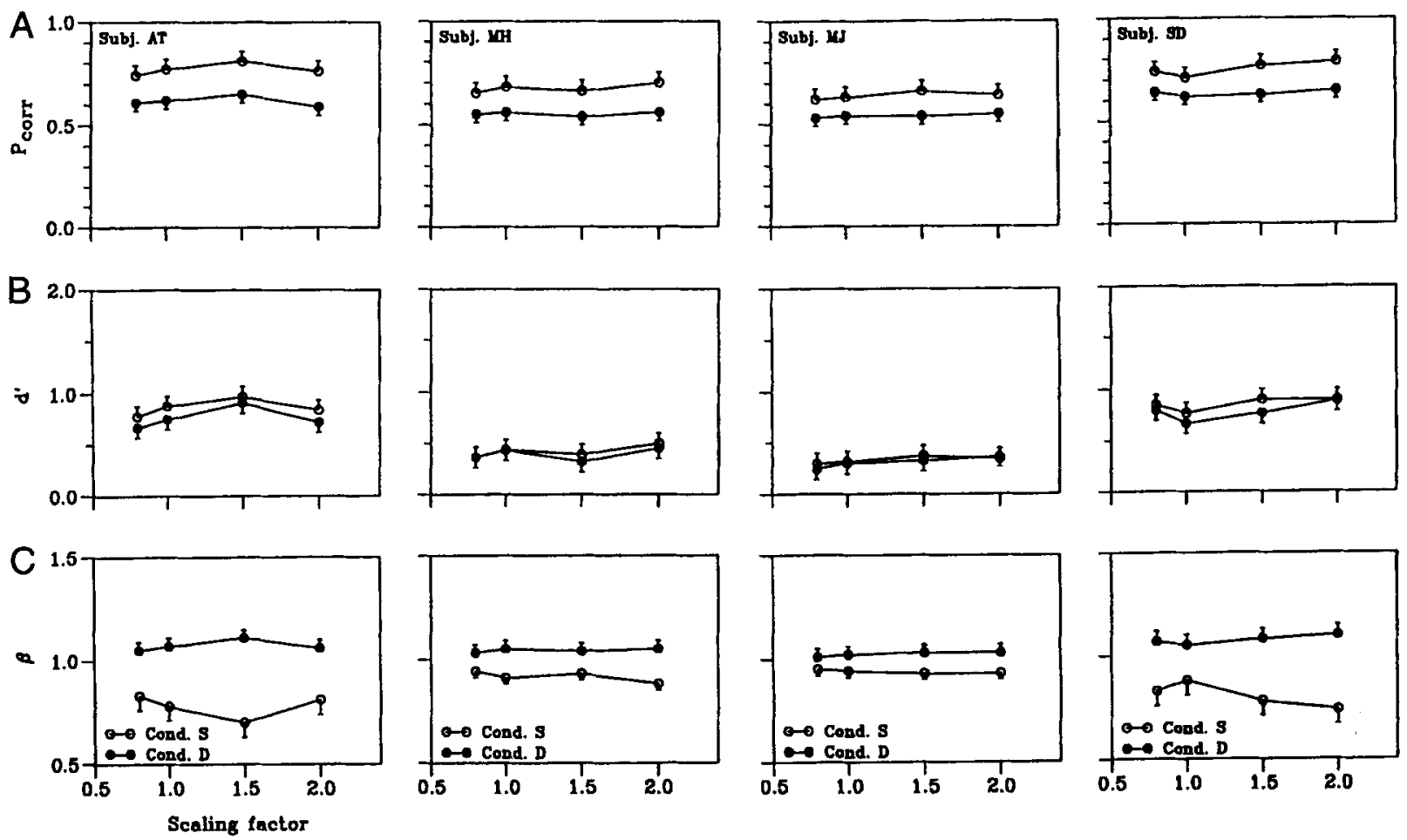

Figure 3. (A) Relative frequency for a correct identification of the test signal as a function of the size (scaling factor) of the presaccadic signal (Experiment 1). Condition $S$ refers to the pooled data of all presentations, where the orientation of the pre- and postsaccadic signal was the same, Condition $D$ to the case of different orientations. The error bars represent $\pm 1 S E$. (B) $d^{\prime}$ values resulting from the signal detection analysis of the relative frequencies shown in (A). (C) $\beta$ values resulting from the signal detection analysis of the relative frequencies shown in (A).

sis of the response data. For two observers (A.T. and S.D.), the $d^{\prime}$ mean values across the range of scale factors reveal a small deterioration of Response Class D with respect to Class $\mathrm{S}$, which in the case of A.T. is significant $[t(6)=2.85, p<.05]$ and is approaching significance for S.D. $[t(6)=2.0, p<.10]$. For the other two observers, there are obviously no significant changes. In contrast to the small differences between Conditions $S$ and $D$ concerning $d^{\prime}$, the associated response criteria $\beta$ diverge distinctly: For Response Class D, we find $\beta$ values below unity, whereas for Response Class $\mathrm{S}$, all $\beta$ values lie above unity. Since $\beta=1$ corresponds to a neutral decision behavior, it can be said that transsaccadic unaltered signals are identified on a low level of redundancy (i.e., $\beta<1$ ), whereas the recognition of transsaccadic-modified signals depends on highly redundant, unequivocal sensory events (i.e., $\beta>1$ ).

The results of Experiment 2 are summarized in Figure 4 . Figure $4 \mathrm{~A}$ gives the percentage correct values for a correct identification of the test stimulus as a function of the position of the presaccadic prime signal. Negative coordinate values correspond to positions to the left of the screen center, and positive values correspond to positions to the right of the screen center. Note that only the position of the presaccadic stimulus was varied, whereas the saccade metrics - that is, the initial fixation point at the screen center (eccentricity $0.0^{\circ}$ ) and the position of the test signal and therefore that of the saccade target (eccentricity $3.0^{\circ}$ )-remained unaltered. The data reveal that the transsaccadic matching effect, characterized by the lower performance index of Response Class $D$ with respect to that of Class $S$, is present over nearly the full range of prime signal positions. This is surprising in particular for Position $-3.0^{\circ}$, which refers to the case in which the prime signal appeared on the contralateral side of where the saccade actually was directed and the test signal was presented $\left(+3.0^{\circ}\right)$. The effect declines only for the most eccentric location $\left(4.5^{\circ}\right)$, which is plausible because vision at such eccentricities is known to be increasingly impaired with respect to both spatial resolution and contrast sensitivity (Hilz \& Cavonius, 1974; Westheimer, 1982). Again, the SDT analysis of the results depicted in Figures 4B and 4C reveals that the difference between Response Classes $\mathrm{S}$ and $\mathrm{D}$ becomes evident in the different values of the Response Criterion $\beta$, whereas the $d^{\prime}$ values do not differ significantly.

\section{EXPERIMENTS 3 AND 4}

Experiments 1 and 2 yielded a high degree of invariance of the transsaccadic matching processes against spatial decorrelations of pre- and postsaccadic stimulus displays caused by either rescaling or shifting operations. In particular, the surprising robustness against lateral shifts of the prime signal suggests an explanation involving attentional processes in the following way: Temporarily, 

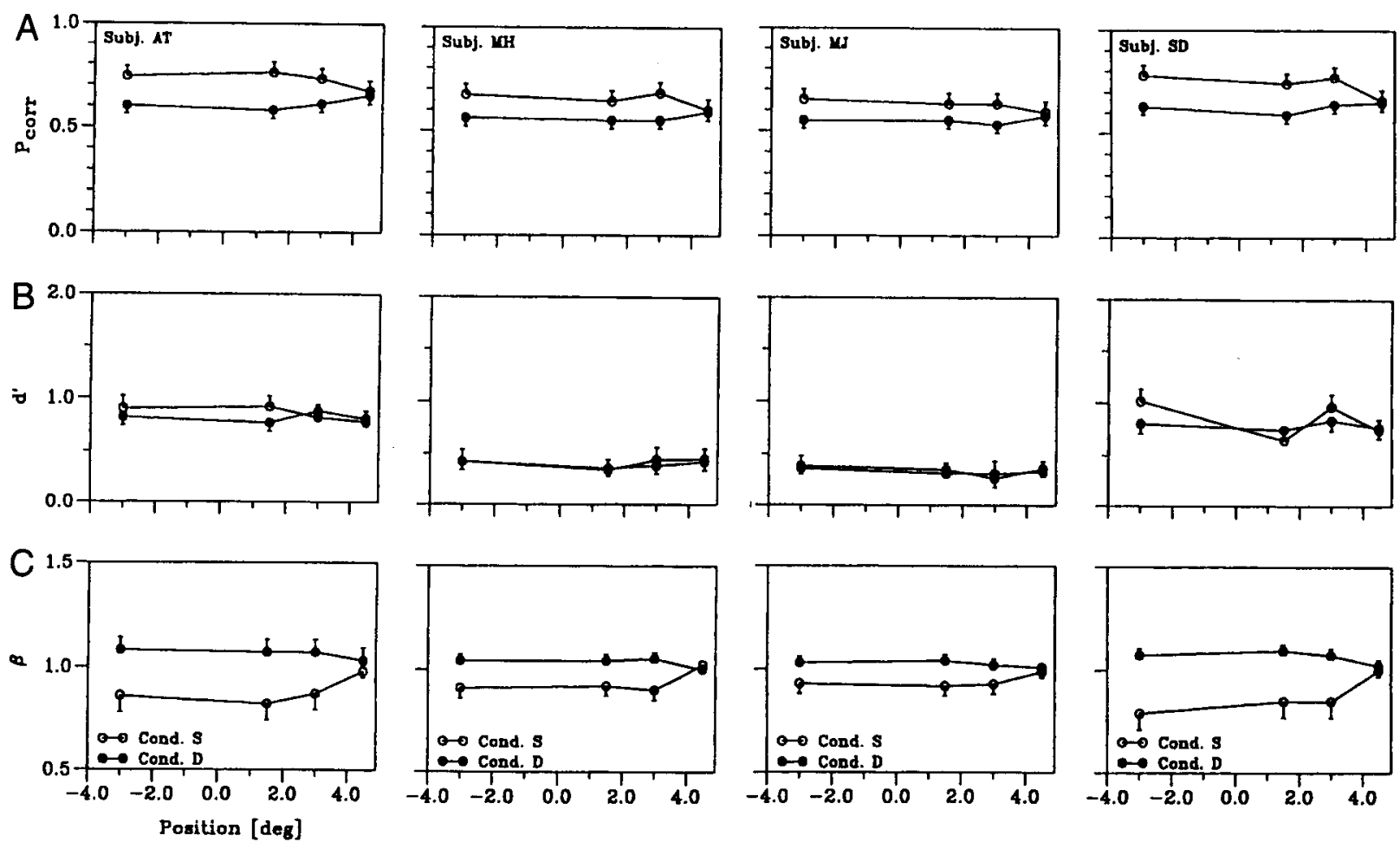

Figure 4. (A) Relative frequency for a correct identification of the test signal as a function of the position of the presaccadic signal (Experiment 2). Negative (positive) values refer to positions to the left (right) of the screen center. Condition $\mathrm{S}$ refers to the pooled data of all presentations, where the orientation of the pre- and postsaccadic signal was the same, Condition $D$ to the case of different orientations. The error bars represent $\pm 1 S E$. (B) $d^{\prime}$ values resulting from the signal detection analysis of the relative frequencies shown in $(A)$. (C) $\beta$ values resulting from the signal detection analysis of the relative frequencies shown in $(A)$.

each trial may be divided into three intervals, the presaccadic interval, the execution of the saccade, and the postsaccadic interval. During the presaccadic period, the subject is fixating the fixation marker in the center of the screen. But gaze direction and direction of visual attention are known to not necessarily coincide with each other. Rather, it is conceivable that despite the stationary eyes, the focus of visual attention will be attracted by the prime signal since this is the only distinctive stimulus on the display. In the next period, during the preparation of the saccade, attention will inevitably be directed to the location of the saccade goal, a notion common for current models of saccade programming (e.g., Becker \& Jürgens, 1979; Fischer, 1987; Rizzolati, Riggio, Dascola, \& Umiltà, 1987; Shepherd, Findlay, \& Hockey, 1986). Finally, the postsaccadically presented test signal will be identified under consideration of presaccadically acquired stimulus information. According to this explanation, the direction of visual attention would provide the spatial correspondence between pre- and postsaccadic processed input.

This hypothesis, if correct, would predict that if the presaccadic display consists of, say, two stimuli of equal salience - a primary signal at the location of the saccade goal and a secondary one acting as distractor at a nontarget position-transsaccadic matching should occur predominantly between the primary signal and the test signal. This is because the primary signal is the one attended to immediately before the saccade starts. We tested this prediction by extending the presaccadic single-ring display originally used in Experiments 1 and 2 to a double-ring display in which the two Landolt rings were placed either symmetrically on both sides of the original fixation point (Experiment 3 ) or on the same side (Experiment 4).

\section{Method}

Subjects, stimuli, and data analysis were the same as those in Experiments 1 and 2. However, the presaccadic stimulus display in Experiments 3 and 4 now consisted of two Landolt rings (Figure 5). In Experiment 3, they were placed symmetrically to the left and to the right of the initial fixation dot at eccentricities of $\pm 3^{\circ}$. The subject had to saccade either to the left or to the right prime signal. The actual direction was indicated in advance by an additional cue in the center of either the left or the right prime signal. The cued prime signal will be referred to as the primary signal, and the uncued prime signal as the secondary or distractor stimulus. The temporal parameters of the stimulus sequence were the same as those in Experiments 1 and 2 . The simultaneous extinction of the prime signals and the displacement of the fixation dot to the cued position triggered the execution of the saccade, followed by the presentation of the test signal at the location of the saccade goal. Note that the test signal was always thus presented at the same spatial location as the presaccadic primary signal. 


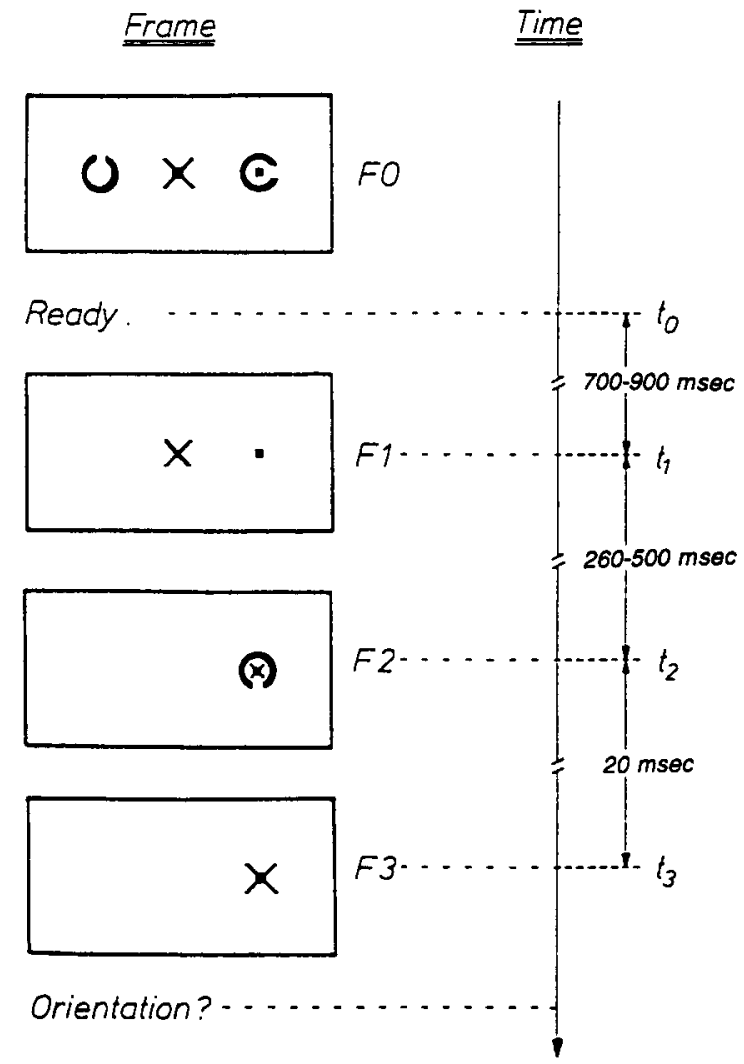

Figure 5. Stimulus sequence of Experiment 3. Here two Landolt rings were placed symmetrically to the left and to the right of the initial fixation point at $3^{\circ}$ eccentricity. One of these signals (here, the right one) is marked by an additional dot as the primary signal - that is, as the target for the saccade to come. The rest of the sequence is identical to that shown in Figure 2. Note, however, that the postsaccadic test signal is always presented at the location of the saccade goal.

For Experiment 4, both prime signals were placed to the right of the screen center with eccentricities of $1.5^{\circ}$ and $3^{\circ}$, respectively. They will be referred to as the "near" and the "far" stimuli. This spatial arrangement was chosen to ensure that the identification of the far signal was not affected by masking effects of the near Landolt ring. Again, two saccades could be triggered either to the near or to the far signal depending on which one was marked as the primary signal (i.e., the saccade target). Again, the test signal was always presented at the location of this primary stimulus.

Saccades to complex structures are known to reveal a center-ofgravity effect (Coren \& Hoenig, 1972; Findlay, 1982) - that is, their distribution of landing points is shifted toward the mean position of the luminance profile, in the present case corresponding to the middle between the two prime signals. Therefore, each saccade was checked for correct amplitude and trials with position errors of more than $0.3^{\circ}$ were rejected and repeated later.

Experiments 3 and 4 were also determined by three parameters, namely the orientation of the presaccadic target signal, the orientation of the presaccadic nontarget signal, and the orientation of the test stimulus. Thus the total number of stimulus configurations amounted to $4 \times 4 \times 4=64$. The experiments were blocked into 320 trial sessions yielding five presentations per configuration. The data from 10 sessions were pooled for a database of 50 presentations per configuration.

\section{Results}

The results of Experiments 3 and 4 are displayed in Figures 6 and 7. Figures $6 \mathrm{~A}$ and $6 \mathrm{~B}$ show the relative frequencies of a correct identification of the test signal for Response Classes S and D, separated into left- and rightdirected saccades, respectively. Since all subjects revealed the same pattern of results, the data are averaged across the 4 observers. The figure shows that the primary signal that spatially coincides with the saccade target influences the classification of the test stimulus much more than does the secondary signal presented on the contralateral side: In the case of the left-directed saccade, transsaccadic matching involves the left prime signal (Figure $6 \mathrm{~A}$ ), whereas in case of right-directed saccades, it is the right prime signal that is matched with the postsaccadic test signal (Figure 6B). Again, this pattern of results is reflected in the corresponding $\beta$ values from signal detection analysis (Table 1).

Figure 7 and Table 2 show the results of Experiment 4. Here the emerging pattern is not as clear-cut as in Experiment 3 . In the case of $1.5^{\circ}$ saccades (Figure 7A), transsaccadic matching occurs between the near prime signal and the test stimulus, as predicted by the above hypothesis, but this also holds for the $3^{\circ}$ saccades (Figure 7B) contrary to what was expected. A possible explanation for this outcome is that although provisions were made to avoid lateral masking of the far Landolt ring by the near one, there still could have been different degrees of salience between both stimuli. As a consequence, visual attention would involuntarily be more attracted by the near prime signal. We will discuss this point further in the Discussion.

\section{DISCUSSION}

So far, the experiments have yielded two main results: First, the analysis of the data in terms of SDT revealed that transsaccadic matching is primarily expressed by a change of criterion and therefore may be related to processes of signal evaluation. Second, concerning the spatial constraints of the matching effect, it was found that these strongly depend on the structuring of the presaccadic display. In the single-prime signal conditions of Experiments 1 and 2, transsaccadic matching exhibited a considerable degree of invariance in relation to spatial decorrelations - that is, the effect did not depend on the degree of spatial overlap between pre- and postsaccadic stimuli. The invariance appeared to be broken or at least reduced in the double-ring situations of Experiment 3 and 4 . This elementary structuring of the presaccadic display led to a preferred matching of the postsaccadic test signal with the primary signal-that is, the prime signal that was shown at the saccade goal.

These results raise the question of whether and to what extent transsaccadic matching is phenomenologically linked to the saccade. For this reason we performed a temporal analysis on the data in which the response data were 
A

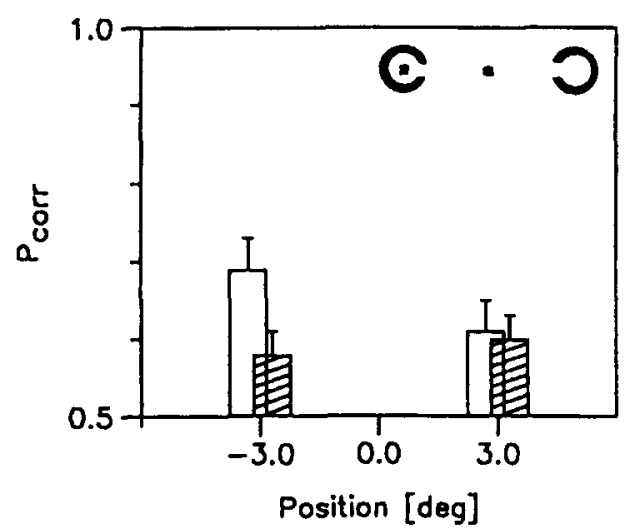

B

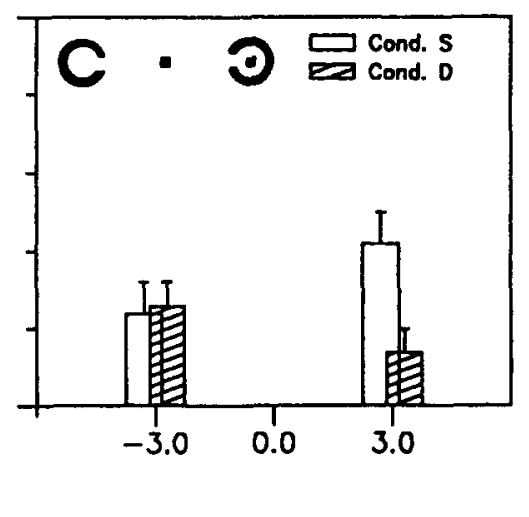

Figure 6. Relative frequencies of a correct identification of the test signal for Response Classes $S$ and $D$ as a function of prime signal position, separated into left- $(A)$ and right- $(B)$ directed saccades. The plot reveals that the primary signal-that is, the presaccadic prime signal at the saccade goal (at $-3^{\circ}$ for left-directed saccades, and at $+3^{\circ}$ for right-directed saccades) - influences the classification of the test stimulus much more than does the secondary signal acting as a presaccadic distractor stimulus on the contralateral side of the visual field.

A

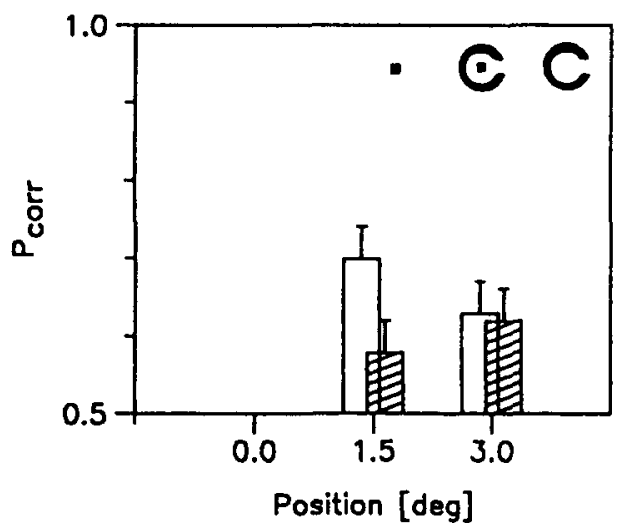

B

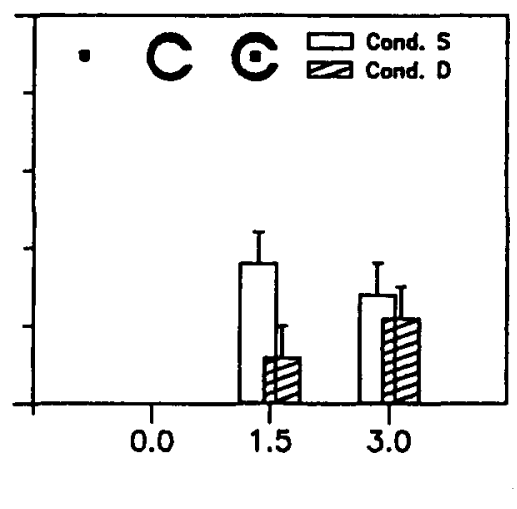

Figure 7. Relative frequencies of a correct identification of the test signal for Response Classes $S$ and D, separated into (A) "near" (target location: $1.5^{\circ}$ ) and (B) "far" (target location: $3.0^{\circ}$ ) saccades. In contrast to Figure 6, the near target always dominates the classification of the test signal, regardless of whether it is acting as primary or secondary stimulus.

related to the stimulus onset asynchrony (SOA) of the presentation of the test stimulus with respect to the termination of the eye movement. Note that the SOA is statistically distributed because of the intrinsic fluctuations of the saccadic latency per se and the additional variation introduced by the randomization of the ISI between pre- and postsaccadic stimulus displays. The resulting effective SOA values covered a range from approximately 80 to $230 \mathrm{msec}$. Response data of the $\mathrm{S}$ and $\mathrm{D}$ groups were pooled across observers in successive temporal SOA intervals of $40 \mathrm{msec}$ bin width and percentage correct; $d^{\prime}$ and $\beta$ values were calculated for each interval separately. This analysis was performed for the data from Experiments 3 and 4. Since both experiments produced similar results, only the data from Experiment 3 are presented in Figure 8.
Two aspects are remarkable in this plot. First, transsaccadic matching as characterized by an S versus D dissociation of percentage correct and $\beta$ values appears to be restricted to a relatively short postsaccadic period, extending to approximately $160 \mathrm{msec}$ after eye movement termination. This means that the phenomenon is temporally linked to the execution of the eye movement, a result that extends Jüttner and Röhler's (1993) observation that this effect depends on the execution of the saccade per se and cannot be elicited by mimicking the corresponding retinal stimulus sequence with stationary eyes.

Second, Figure 8 reveals that in addition to the dissociation of the Response Criterion $\beta$ for the Response Groups $\mathrm{S}$ and $\mathrm{D}$ there is also a small decrease in $d^{\prime}$ affecting both groups simultaneously. Note that this change is 


\begin{tabular}{|c|c|c|c|c|}
\hline \multirow[b]{4}{*}{ Condition } & $\begin{array}{c}\text { Corred } \\
\text { ecision } \\
\text { Obse }\end{array}$ & $\begin{array}{l}\text { ble1 } \\
\text { es } \overline{p_{\text {corr }}}, \\
\text { ion } \overline{\boldsymbol{\beta}} \mathrm{A} \\
\text { Exper }\end{array}$ & $\begin{array}{l}\text { ivity In } \\
\text { d Acro } \\
3\end{array}$ & \\
\hline & \multicolumn{4}{|c|}{ Saccade Target Position } \\
\hline & \multicolumn{2}{|c|}{$\begin{array}{c}-3^{\circ} \\
\text { Prime Signal Position } \\
\end{array}$} & \multicolumn{2}{|c|}{$\begin{array}{c}3^{\circ} \\
\text { Prime Signal Position } \\
\end{array}$} \\
\hline & $-3^{\circ}$ & $3^{\circ}$ & $-3^{\circ}$ & $3^{\circ}$ \\
\hline \multicolumn{5}{|c|}{$\overline{p_{c o r r}}(S E<.04)$} \\
\hline S & 0.69 & 0.61 & 0.62 & 0.71 \\
\hline D & 0.58 & 0.60 & 0.63 & 0.57 \\
\hline \multicolumn{5}{|c|}{$\overline{d^{\prime}}(S E<.06)$} \\
\hline S & 0.54 & 0.51 & 0.58 & 0.53 \\
\hline $\mathrm{D}$ & 0.51 & 0.50 & 0.59 & 0.50 \\
\hline \multicolumn{5}{|c|}{$\bar{\beta}(S E<.03)$} \\
\hline S & 0.90 & 0.99 & 1.01 & 0.87 \\
\hline $\mathrm{D}$ & 1.04 & 1.00 & 1.00 & 1.04 \\
\hline
\end{tabular}

masked in Figures 3 and 4 and Tables 1 and 2 because of the pooling across the entire SOA continuum. Hence both the $d^{\prime}$ and $\beta$ values in these figures are to be interpreted as temporal averages weighted by the relative response frequency within the temporal domain. Therefore, the variation of $\beta$ in Figures 3 and 4 appears slightly reduced in comparison to the extrema in Figure 8. Similarly, the $d^{\prime}$ values lie in between the range of those observed in Figure 8.

Whereas the $d^{\prime}$ effect may be attributed to a residual (post-) saccadic suppression known to become manifest also in a decline of sensitivity in terms of signal detection analysis (Pearce \& Porter, 1970), the dissociation of the Criterion $\beta$ may indicate some sort of selective processing of postsaccadic sensory information that depends on the context of the presaccadic stimulus display. Before discussing the relevance of this effect for the problem of visual stability, I offer the following caveat. At the outset it should be noted that the problem of visual stability has a long scientific history (Grüsser, 1986) and, in its most general formulation, involves several subsystems and a great number of cues (for reviews, see Bischof \& Kramer, 1968; Bridgeman, Van der Heijden, \& Velichkovsky, 1994; MacKay, 1973; Shebilske, 1977). Here I will focus primarily on one aspect, namely the question of why and what sort of visual information has to be preserved across saccades.

One of the most recent suggestions for solving the problem of visual stability in the context of saccadic eye movements is the calibration solution of Bridgeman et al. (1994). Basic to their approach is that it regards retinotopic representations as sufficient to ensure visual stability. This notion is based on the distinction between where something is coded in the brain and how something is coded —or, more specifically, on the fact that the cortical position does not necessarily code position of objects in the world. Accordingly, if the pattern of activation is displaced in retinotopic maps as the result of a saccade, this change does not encode a displacement. Moreover, whereas the retinotopic position may change, the activation pattern itself remains constant, and the perceived position may be retained by recalibrating this representation anew with each fixation by means of a position signal originating from different retinal and/or extraretinal sources. The calibration solution therefore reduces perceptual constancy across saccades to the constancy of the neural activation pattern in the various retinotopic representations, whereas direction constancy is maintained via a process of recalibration acting upon these representations.

For the moment, that approach appears not to require any transsaccadic processing of pictorial information at all. This is because the given constancy of the visual input is identified with a putative constancy of its corresponding retinotopic representations that makes transsaccadic matching dispensable. But the latter assumption again raises problems because of the radial anisotropy of the visual field. As noted in the introduction, there is evidence (Jüttner \& Rentschler, 1996; Rentschler et al., 1994; Strasburger et al., 1994) that internal representations of foveal and extrafoveal differ substantially from each other in terms of their perceptual dimensionality. The object in the periphery becoming a target for a saccadic eye movement and the object fixated after termination of the saccade may be the same, but the corresponding neural activation patterns are not on account of this foveal-extrafoveal incompatibility. In other words, there may be a form of semantic constancy concerning both objects rather than one concerning their retinotopic or otherwise early representations. But the former has to be asserted in an additional step, which means that the original calibration solution has to be extended by a process of semantic evaluation bridging the saccade.

At this point the functional role of transsaccadic visual matching becomes apparent, since the underlying process can be characterized in this very sense - namely as a semantic evaluation of the newly fixated object. The probing of the observer with a semantically ambivalent signal after termination of the saccade indicates that this signal is identified according to what has been seen at the same location of the visual field before the eyes

Table 2

Percentage Correct Values $\overline{p_{\text {corr }}}$, Sensitivity Index $\overline{d^{\prime}}$, and Decision Criterion $\widetilde{\beta}$, Averaged Across Observers in Experiment 4

\begin{tabular}{|c|c|c|c|c|}
\hline \multirow[b]{3}{*}{ Condition } & \multicolumn{4}{|c|}{ Saccade Target Position } \\
\hline & \multicolumn{2}{|c|}{$\begin{array}{c}1.5^{\circ} \\
\text { Prime Signal Position } \\
\end{array}$} & \multicolumn{2}{|c|}{$\begin{array}{c}3^{\circ} \\
\text { Prime Signal Position }\end{array}$} \\
\hline & $1.5^{\circ}$ & $3^{\circ}$ & $1.5^{\circ}$ & $3^{\circ}$ \\
\hline \multicolumn{5}{|c|}{$\overline{p_{\text {corr }}}(S E<.04)$} \\
\hline S & 0.70 & 0.63 & 0.68 & 0.64 \\
\hline $\mathrm{D}$ & 0.58 & 0.62 & 0.56 & 0.61 \\
\hline \multicolumn{5}{|c|}{$\bar{d}^{\prime}(S E<.06)$} \\
\hline S & 0.52 & 0.55 & 0.52 & 0.58 \\
\hline $\mathrm{D}$ & 0.53 & 0.56 & 0.53 & 0.56 \\
\hline \multicolumn{5}{|c|}{$\bar{\beta}(S E<.03)$} \\
\hline $\mathrm{S}$ & 0.88 & 0.98 & 0.91 & 0.96 \\
\hline $\mathrm{D}$ & 1.03 & 1.01 & 1.02 & 1.01 \\
\hline
\end{tabular}



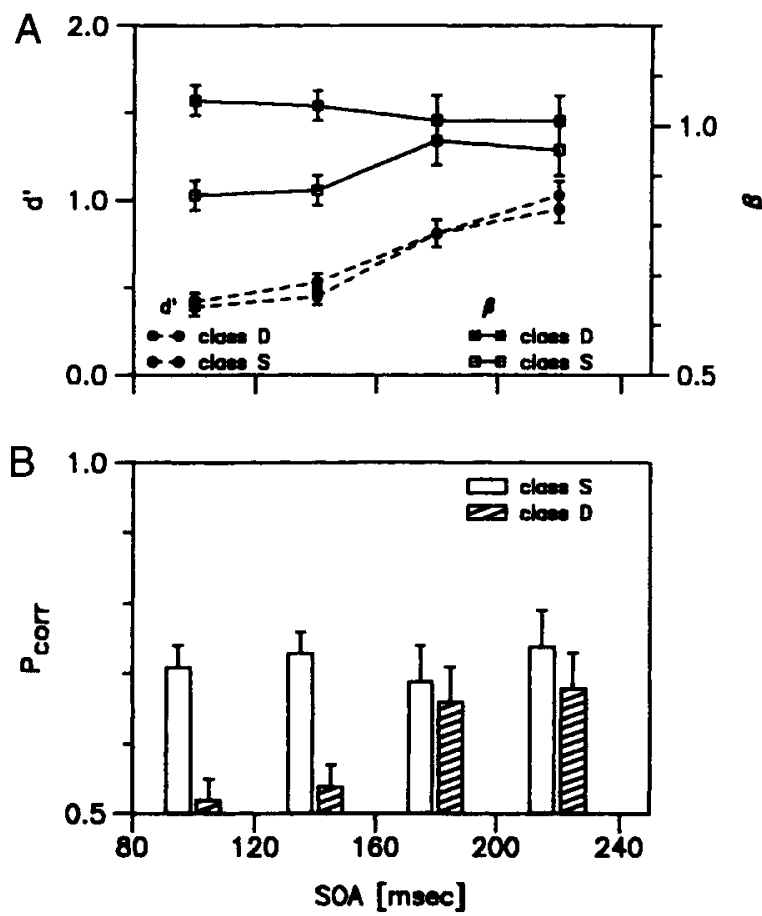

Figure 8. (A) Corresponding sensory $\left(d^{\prime}\right)$ and criterion $(\beta)$ measures of a signal detection analysis of the response data. (B) Relative frequency of a correct identification of the test signal as a function of the stimulus onset asychrony (SOA) between the end of the saccade and the onset of the test stimulus. Response data are pooled within consecutive time intervals of $\mathbf{4 0}$ msec. Transsaccadic matching extends approximately up to $160 \mathrm{msec}$ after saccade termination.

started. To some extent this finding is reminiscent of object-specific priming effects (Kahneman, Treisman, \& Gibbs, 1992) - that is, a preview benefit resulting from the fact that both prime and target are seen as states of the same perceived object. However, the interpretation is not as straightforward as this for two reasons: First, object-specific priming is observed under a variety of conditions - in particular, with stationary eyes. In contrast, transsaccadic matching appears to be phenomenologically tied to the motor execution of a saccade (Jüttner \& Röhler, 1993). Second, object-specific priming bridges temporal gaps between prime and target of at least $600 \mathrm{msec}$. Transsaccadic matching, on the other hand, spans a postsaccadic period of only $160 \mathrm{msec}$. Hence the two processes almost operate on different time scales (10s vs. 100s of milliseconds). Consequently, from a purely phenomenological point of view, the two effects would appear to be relatively independent of each other. This, however, does not rule out the possibility that the two might be indirectly related, in the sense of a sharing of common subprocesses and representations-for instance, in terms of the object files of Kahneman et al. acting as episodic, though not necessarily labeled, representations of "real-world" objects. The difference with respect to the temporal constraints could be due to the different role of attentional processes. Priming effects are known to be influenced by selective attention (Tipper \& Cranston, 1985), whereas transsaccadic matching requires the mediation of involuntary attentional processes, presumably linked to oculomotor programming. I will return to the role of attention later.

Transsaccadic matching could be qualified as a process of context-sensitive evaluation of the postsaccadic signal, in which the context is given by the presaccadic stimulus display. The characterization of this process as "evaluative" appears adequate in light of the signal detection analysis, which revealed that transsaccadic matching manifests in a dissociation of the Decision Criterion $\beta$. The dissociation $(\beta<1$ for identical prime and test signal; $\beta>1$ for different prime and test signal) is such that it results in a suppression of postsaccadic stimulus information that is incompatible with presaccadic processed information. In the past, the notion of a stability criterion was also proposed by MacKay (1973) and MacKay and Mittelstaedt (1974) within the framework of their evaluation hypothesis concerning the problem of visual stability. Their conception is based on the idea of an internal representation of the outside world that is assumed a priori to be stable, and that may be updated by sensory signals. The central role here is played by a process of evaluation comparing the actual visual input and the internal representation to decide whether the latter needs revision. The criteria for this comparison would be continuously varied according to motor activity. In the concrete case of a saccade, this account would predict a conservative system behavior toward intersaccadic stimulus changes.

Although our results are in agreement with this prediction, they also reveal that the evaluation mechanism does not cover the entire visual field, but seems to be restricted to the area of the saccade target. However, the binding of pre- and postsaccadic perception via the saccade target could be found only with a (even elementary) structured visual field (Experiments 3 and 4 ). This strong dependency on the spatial configuration of the presaccadic stimulus display suggests that functionally the influence of perceptual context on transsaccadic matching is mediated by visual attention. On the one hand, visual attention is considered to be one of the prerequisites for the execution of a saccadic eye movement (Fischer, 1987; Rizzolati et al., 1987; Shepherd et al., 1986). On the other hand, in the context of visual search and texture segregation, attention is often interpreted as a localization mechanism for texture elements (Julesz, 1981) or features (Treisman \& Gelade, 1980). The presaccadic shift of visual attention therefore could be conceived as a process that defines a certain region centered around the saccade goal, the content of which is postsaccadically matched against the information processed foveally after the eye movement. This means that transsaccadic matching is restricted to a relatively small proportion of the visual field, defined by the attentional window around the saccade goal. Attention therefore provides the correspondence between preview (prime signal) and target (test signal). The predominant role of the saccade target 
has been confirmed by recent observations of Irwin, McConkie, Carlson-Radvensky, and Currie (1994), according to which spatial stability of the saccade target is the prerequisite for the perception of visual stability. Furthermore, it is compatible with the notion of a combination of successively fixated parts of the visual field that form the basis for spatial representations (Hayhoe et al., 1991).

The participation of attentional mechanisms would explain the differences between the results of Experiments 1 and 2 on the one hand, and of Experiments 3 and 4 on the other, since the spatial distribution of attention is known to depend on a variety of different factors concerning task and precuing (see Posner, 1980; Shulman, Remington, \& McLean, 1979). Moreover, concerning the dynamics of visual attention, it has been found that with peripheral targets, the attentional field first covers the whole relevant hemifield before focusing on the actual target (Erikson \& Yeh, 1985; Shepherd \& Müller, 1989). This might explain why in Experiment 3 a clear dominance of the primary presaccadic signal located in the hemifield of the actual saccade target was obtained, whereas the results were much less clear in Experiment 4, in which both presaccadic signals appeared in the same hemifield and the attentional field might not have narrowed sufficiently to separate them.

Transsaccadic visual matching, then, appears to be a phenomenon that may contribute to maintaining visual stability in matching the attended target of the saccade with the postsaccadic fixated target on a semantic level of representation. In the present experiments, it has been shown that conceptionally this may be regarded as an extension of the calibration theory of Bridgeman et al. (1994), enforced by the radial anisotropy of the visual field. Alternatively, it may be considered as a contextsensitive evaluation theory in the sense of MacKay (1973): the context is provided by the saccade target attended to immediately before the eyes start to move. In both cases, transsaccadic matching acts in the sense of a conservation of current perception. In this respect it appears as the cognitive counterpart of the well-known mechanism of spatial suppression concerning intrasaccadic stimulus displacements (Bridgeman et al., 1975).

\section{REFERENCES}

BECKER, W., \& JÜrGENS, R. (1979). An analysis of the saccadic system by means of double step stimuli. Vision Research, 19, 967-983.

Bischof, N., \& Kramer, E. (1968). Untersuchungen und Überlegungen zur Richtungswahrnehmung bei willkürlichen sakkadischen Augenbewegungen [Investigations and reflections on perceived direction during voluntary saccadic eye movements]. Psychologische Forschung, 32, 185-218.

Breitmeier, B. G., Kropfl, W., \& Julesz, B. (1982). The existence and role of retinotopic and spatiotopic forms of visual persistence. Acta Psychologica, 52, 175-196.

BRIDGEMAN, B. (1983). Mechanisms of space constancy. In A. Hein \& M. Jeannerod (Eds.), Spatially oriented behaviour (pp. 263-279). Berlin: Springer.

Bridgeman, B., Hendry, D., \& Stark, L. (1975). Failure to detect displacement of the visual world during saccadic eye movements. $\mathrm{Vi}$ sion Research, 15, 719-722.
Bridgeman, B., \& MAYER, M. (1983). Failure to integrate visual information from successive fixations. Bulletin of the Psychonomic Society, 21, 285-286.

Bridgeman, B., Van der Heijden, A. H. C., \& Velichkovsky, B. M. (1994). A theory of visual stability across saccadic eye movements. Behavioral \& Brain Sciences, 17, 247-292.

Coren, S., \& Hoenig, P. (1972). Effect of non target stimuli upon the length of voluntary saccades. Perceptual \& Motor Skills, 34, 499-508.

ERIKSON, C. W., \& YEH, Y. Y. (1985). Allocation of attention in the visual field. Journal of Experimental Psychology: Human Perception \& Performance, 11, 583-597.

FeldMAN, J. (1985). Four frames suffice: A provisional model of vision and space. Behavioral \& Brain Sciences, 8, 265-289.

FindLAY, J. M. (1982). Global visual processing for saccadic eye movements. Vision Research, 22, 1033-1045.

Fischer, B. (1987). The preparation of visually guided saccades. Reviews of Physiology, Biochemistry \& Pharmacology, 106, 1-35.

GERBER, K. (1987). Wechselwirkungen von globalen und lokalen Bewegungsreizen im visuellen System des Menschen. Unpublished doctoral dissertation, University of Munich.

GREEN, D. M. \& SwETS, J. M. (1974). Signal detection theory \& psychophysics. New York: Krieger.

GRÜSSER, O.-J. (1986). Interaction of efferent and afferent signals in visual perception. A history of ideas and experimental paradigms. Acta Psychologica, 63, 3-21.

Hayhoe, M., Lachter, J., \& Feldman, J. (1991). Integration of form across saccadic eye movements. Perception, 20, 393-402.

Hilz, R., \& Cavonius, C. R. (1974). Functional organization of the peripheral retina: Sensitivity to periodic stimuli. Vision Research, 14, 1333-1337.

IRWIN, D. E. (1992). Perceiving an integrated visual world. In D. E. Meyer \& S. Kornblum (Eds.), Attention and performance XIV: Synergies in experimental psychology, artificial intelligence, and cognitive neuroscience (pp. 121-142). Hillsdale, NJ: Erlbaum.

Irwin, D. E., McConkie, G. W., Carlson-Radvensky, L. A., \& CurRIE, C. (1994). A localist evaluation solution for visual stability across saccades. Behavioral \& Brain Sciences, 17, 265-266.

IRWIN, D.E., YANTIS, S., \& JoNides, J. (1983). Evidence against visual integration across saccadic eye movements. Perception \& Psychophysics, 34, 49-57.

JoNides, J., IRWIN, D. E., \& YanTIS, S. (1983). Failure to integrate information from successive fixations. Science, 222, 188.

JULESZ, B. (1981). Textons, the elements of texture perception and their interactions. Nature, 290, 91-97.

JÜTtNer, M., \& RentschleR, I. (1996). Reduced perceptual dimensionality in extrafoveal vision. Vision Research, 36, 1007-1022.

JÜTt NER, M., \& RöHLER, R. (1993). Lateral information transfer across saccadic eye movements. Perception \& Psychophysics, 53, 210-220.

Kahneman, D., Treisman, A., \& GibBs, B. J. (1992). The reviewing of object files: Object-specific integration of information. Cognitive Psychology, 24, 175-219.

Koenderink, J. J., Bouman, M. A., Bueno de Mesquita, A. E., \& SLAPPENDEL, S. (1978). Perimetry of contrast detection thresholds of moving spatial sine wave patterns: III. The target extent as a sensitivity controlling parameter. Journal of the Optical Society of America $A, 68,854-860$.

MACKAY, D. M. (1973). Visual stability and voluntary eye movements. In R. Jung (Ed.), Handbook of sensory physiology (Vol. 7, pp. 307331). Berlin: Springer.

MacKay, D. M., \& Mittelstaedt, H. (1974). Visual stability and motor control (reafference revisited). In W. D. Keidel, W. Händler, \& M. Spreng (Eds.), Kybernetik und Bionik (pp. 71-80). Munich: Oldenbourg.

McConkIE, G. W., \& ZoLA, D. (1979). Is visual information integrated across successive fixations in reading? Perception \& Psychophysics, 25, 221-224.

Pearce, D., \& Porter, E. (1970). Changes in visual sensitivity associated with voluntary saccades. Psychonomic Science, 19, 225-227.

Pollatsek, A., Rayner, K., \& Collins, W. (1984). Integrating pictorial information across eye movements. Journal of Experimental $P_{s y-}$ chology: General, 113, 426-442.

Pollatsek, A., Rayner, K., \& Henderson, J. M. (1990). Role of spa- 
tial location in integration of pictorial information across saccades. Journal of Experimental Psychology: Human Perception \& Performance, 16, 199-210.

Posner, M. I. (1980). Orienting of attention. Quarterly Journal of Experimental Psychology, 32, 3-25.

RAYNER, K., MCCONKIE, G. W., \& Zola, D. (1980). Integrating information across eye movements. Cognitive Psychology, 12, 206-226.

RaYNer, K., \& Pollatsek, A. (1983). Is visual information integrated across saccades? Perception \& Psychophysics, 34, 39-48.

RentsChler, I., JÜtTNer, M., \& CAELlI, T. (1994). Probabilistic analysis of human supervised learning and classification. Vision Research, 34, 669-687.

Riggs, L. A., Merton, P. A., \& Morton, H. B. (1974). Suppression of visual phosphenes during saccadic eye movements. Vision Research, 14, 997-1011.

Rizzolati, G., Riggio, L., Dascola, 1., \& Umiltà, C. (1987). Reorienting attention across the horizontal and vertical meridians: Evidence in favour of a premotor theory of attention. Neuropsychologia, 25, 31-40.

Rovamo, J., \& VIRSU, V. (1979). An estimation and application of the human cortical magnification factor. Experimental Brain Research, 37, 495-510.

ShEBILSKE, W. L. (1977). Visuomotor coordination, visual direction and position constancies. In W. Epstein (Ed.), Stability and constancy in visual perception: Mechanisms and processes (pp. 23-63). New York: Wiley.

SHEPHERD, M., FINDLAY, J. M., \& HoCKEY, R. J. (1986). The relation- ship between eye movements and spatial attention. Quarterly Journal of Experimental Psychology, 38, 475-491.

SHEPHERD, M., \& Müller, H. J. (1989). Movement versus focusing of visual attention. Perception \& Psychophysics, 46, 146-154.

Shulman, G. L., Remington, R. W., \& McLean, J. P. (1979). Moving attention through visual space. Journal of Experimental Psychology. Human Perception \& Performance, 5, 522-526.

SKOLNIK, M. I. (1970). Radar handbook. New York: McGraw-Hill.

Strasburger, H., Harvey, L. O., Jr., \& Rentschler, I. (1991). Contrast thresholds for identification of numeric characters in direct and eccentric view. Perception \& Psychophysics, 49, 495-508.

Strasburger, H., Rentschler, I., \& Harvey, L. O., JR. (1994). Cortical magnification theory fails to predict visual recognition. European Journal of Neuroscience, 6, 1583-1588.

TIPPER, S. P., \& Cranston, M. (1985). Selective attention and priming: Inhibitory and facilitatory effects of ignored primes. Quarterly Journal of Experimental Psychology, 37A, 591-611.

Treisman, A. M., \& Gelade, G. (1980). A feature-integration theory of attention. Cognitive Psychology, 12, 97-136.

Volkman, F., Schick, A., \& Riggs, L. (1968). Time course of visual inhibition during voluntary saccades. Journal of the Optical Society of America, 58, 562-569.

WESTHEIMER, G. (1982). The spatial grain of the perifoveal visual field. Vision Research, 22, 157-162.

(Manuscript received January 17, 1995; revision accepted for publication May $21,1996$. 